\title{
Blow-up of solutions for the heat equations with variable source on graphs
}

Qiao $\mathrm{Xin}^{\mathrm{a}, *}$, Dengming Liu ${ }^{\mathrm{b}}$

${ }^{a}$ College of Mathematics and Statistics, Yili Normal University, 835000 Yining, P. R. China.

${ }^{b}$ School of Mathematics and Computational Science, Hunan University of Science and Technology, 411201 Xiangtan, P. R. China.

Communicated by Y. J. Cho

\begin{abstract}
In this paper, we mainly consider the blow-up problem for the discrete heat equations with variable source on finite graphs

$$
u_{t}=\Delta_{\omega} u+u^{p(x)}
$$

with homogeneous Dirichlet boundary conditions and positive initial energy. We prove that the corresponding solutions blow up at a finite time with large enough initial data. Moreover, the blow-up rate is also considered. (c) 2016 All rights reserved.
\end{abstract}

Keywords: Blow-up, discrete heat equation, variable reaction, finite graphs.

2010 MSC: 35B44, 35R02, 35B40.

\section{Introduction}

Let $G$ be a graph with vertex set $V$ and edge set $E$, where the vertex set is divided into the boundary vertices $\partial S$ and the interior vertices $S$ which is connected and we always assume $G$ is a finite, connected and simple (without multiple edges and loops) graph in the following context. In this paper, we will consider the following discrete semi-linear parabolic problem on graphs

$$
\begin{cases}u_{t}(x, t)=\Delta_{\omega} u(x, t)+u^{p(x)}(x, t), & x \in S, t \in(0,+\infty), \\ u(x, t)=0, & x \in \partial S, t \in(0,+\infty), \\ u(x, 0)=u_{0}(x) \geq 0, & x \in S\end{cases}
$$

\footnotetext{
* Corresponding author

Email addresses: xinqiaoylsy@163.com (Qiao Xin), liudengming08@163.com (Dengming Liu)
} 
where the function $p(x): S \rightarrow(0,+\infty)$ and $p^{-}:=\min _{x \in S} p(x), p^{+}:=\max _{x \in S} p(x) . C(V)$ denotes the set of all functions which are definite on the vertices $V$ of the graph $G$ and $\Delta_{\omega}$ denotes the $\omega$-Laplacian operator, which is defined as follows:

$$
\Delta_{\omega} u(x)=\sum_{y \in V}[u(y)-u(x)] \omega(x, y),
$$

where the function $\omega(x, y)$ is called the weighted function and satisfies

(i) $\omega(x, x)=0$, for any $x \in V$,

(ii) $\omega(x, y)=\omega(y, x) \geq 0$, for any $x, y \in V$,

(iii) $\omega(x, y)=0$, if and only if $(x, y) \notin E$.

The parabolic equations involving sources like the ones in (1.1) occur in many applied mathematical models, such as heat and energy transfer, electrical networks, image processing and so on [3, 5, 6, 9]. In the continuous case, the following initial boundary value for the semi-linear heat equation

$$
\begin{cases}u_{t}=\Delta u+u^{p(x)}, & x \in \Omega, t \in(0,+\infty), \\ u(x, t)=0, & x \in \partial \Omega, t \in(0,+\infty), \\ u(x, 0)=u_{0}(x) \geq 0, & x \in \Omega\end{cases}
$$

has been considered by many authors, the interested readers can refer to [7, 8, 10] and the references therein. For the discrete case, when $p(x) \equiv p>1$, the Problem (1.1) has been considered in [2, 11, 12]. However, nonconstant powers seem to be new in the literature. In the next section, we will study the local existence of positive solutions and then, the comparison principal to the Problem 1.1 , when $0<p(x) \leq 1$, the solutions to the Problem (1.1) is global by the comparison. The existence of solutions which blows up in finite time for sufficiently large initial data will be studied in the last section.

\section{The local existence of solutions}

Before prove the local existence of solutions, we need some basic knowledge on the heat kernel.

Suppose $\lambda_{1}, \lambda_{2}, \cdots, \lambda_{n}$ are the eigenvalues of the operator $-\Delta_{\omega}$ on $S$ with Dirichlet boundary condition, the corresponding eigenfunctions are denoted by $\phi_{j}(x), j=1,2, \cdots, n$ and satisfy $\sum_{x \in S}\left|\phi_{1}(x)\right|^{2}=1$, where $n=|S|$ is the number of vertices of the interior vertices $S$. Furthermore, set $\lambda_{1}$ be the smallest eigenvalue of $-\Delta_{\omega}$, it is well-known that $\lambda_{1}>0$ and its corresponding eigenfunctions $\phi_{1}(x)$ can be chosen as $\phi_{1}(x)>0$. Its proof can be found in the references [1, 4. And then, the Laplacian operator $-\Delta_{\omega}$ on $S$ with Dirichlet boundary condition can be written as

$$
-\Delta_{\omega}=\sum_{i=1}^{n} \lambda_{i} P_{i}
$$

where $P_{i}$ is the projection of $-\Delta_{\omega}$ to the $i$-th eigenfunction $\phi_{i}$. For any $t \geq 0$, the heat kernel $H_{t}$ of the subgraph $S$ subject to Dirichlet boundary condition is defined to be the $n \times n$ matrix

$$
H_{t}=\sum_{i=1}^{n} e^{-\lambda_{i} t} P_{i}
$$

About the heat kernel, we have the following basic facts:

Lemma 2.1 ([1]). (i) The heat kernel $H_{t}$ satisfies

$$
H_{t}(x, y)=\sum_{i=1}^{n} e^{-\lambda_{i} t} \phi_{i}(x) \phi_{i}(y) .
$$


(ii) For any function $f(x): S \cup \partial S \rightarrow R$, set $F(x, t)=\sum_{y \in V} H_{t}(x, y) f(y)$, we know that $F(x, t)$ satisfies the discrete heat equation with Dirichlet boundary condition and the initial value $f(x)$, i.e.

$$
\begin{cases}F_{t}(x, t)=\Delta_{\omega} F(x, t), & x \in S, t \in(0,+\infty), \\ F(x, t)=0, & x \in \partial S, t \in(0,+\infty), \\ F(x, 0)=f(x) \geq 0, & x \in S\end{cases}
$$

Theorem 2.2. There exists $T>0$ such that the Equation (1.1) has a unique solution in $[0, T]$.

Proof. Now, we begin with proving the local existence in time for the Equation (1.1). Assume $u(x, t)$ is a solution of the Equation (1.1), this leads to $u(x, t)$ satisfies

$$
u(x, t)=\sum_{y \in V} H_{t}(x, y) u_{0}(y)+\int_{0}^{t} \sum_{y \in V} H_{t-s}(x, y) u^{p(y)}(y, s) d s .
$$

Next, we define an inductively sequence $u_{n}(x, t)$ as follows:

$$
\begin{aligned}
u_{1}(x, t) & =0 \\
u_{n+1}(x, t) & =\sum_{y \in V} H_{t}(x, y) u_{0}(y)+\int_{0}^{t} \sum_{y \in V} H_{t-s}(x, y) u_{n}^{p(y)}(y, s) d s .
\end{aligned}
$$

We consider the convergence of the inductively sequence $u_{n}(x, t)$. Set

$$
Q(u)=\int_{0}^{t} \sum_{y \in V} H_{t-s}(x, y) u^{p(y)}(y, s) d s,
$$

we will prove the operator $Q$ is contraction in

$$
E=\left\{u(x, t) \mid u\left(x_{i}, t\right) \in C^{1}[0, T], i=1,2, \cdots, n \text { and }\|u(x, t)\|_{\infty} \leq M\right\},
$$

where $T>0$ is a fixed constant,

$$
\|u(x, t)\|_{\infty}:=\max _{0 \leq t \leq T} \max _{x \in S}|u(x, t)|,
$$

$M$ is also a fixed positive constant and such that $M>\left\|u_{0}(x)\right\|_{\infty}$. For any fixed $x \in S$, by the mean value theorem, we get

$$
u^{p(x)}-v^{p(x)}=p(x)(\theta u+(1-\theta) v)^{p(x)-1}(u-v),
$$

where $\theta(x) \in(0,1)$ for any fixed $x$. Hence, for any $u, v \in E$, we have

$$
\begin{aligned}
\|Q(u)-Q(v)\|_{\infty} & =\left\|\int_{0}^{t} \sum_{y \in V} H_{t-s}(x, y)\left(u^{p(y)}(y, s)-v^{p(y)}(y, s)\right) d s\right\|_{\infty} \\
& =\left\|\int_{0}^{t} \sum_{y \in V} H_{t-s}(x, y) p(y)(\theta u+(1-\theta) v)^{p(y)-1}(u-v) d s\right\|_{\infty} \\
& \leq p^{+}(2 M)^{p^{+}-1} \mu(t)\|u-v\|_{\infty},
\end{aligned}
$$

where $\mu(t)=\sup _{0 \leq t \leq T}\left|\int_{0}^{t} \sum_{y \in V} H_{t-s}(x, y) d s\right|$. On the other hand, we also have $\mu(t) \rightarrow 0$ when $t \rightarrow 0$, so we can choose $T$ is small enough such that $p^{+}(2 M)^{p^{+}-1} \mu(t)<1$ and then, we have $Q$ is contraction. Thus, due to (2.6), we have

$$
\left\|u^{n+1}-u^{n}\right\|_{\infty}=\left\|Q\left(u^{n}\right)-Q\left(u^{n-1}\right)\right\|_{\infty} \leq p^{+}(2 M)^{p^{+}-1} \mu(t)\left\|u^{n}-u^{n-1}\right\|_{\infty} .
$$


So, we have the inductively sequence $u_{n}(x, t)$ is convergent and its limit denotes by $u(x, t)$.

Next, we consider the uniqueness. Suppose $\widetilde{u}(x, t)$ is another solution, it is easy to verify that

$$
\|u-\widetilde{u}\|_{\infty} \leq\left(p^{+}(2 M)^{p^{+}-1} \mu(t)\right)^{n-1}\|u-\widetilde{u}\|_{\infty}
$$

and then, let $n \rightarrow+\infty$ for both sides of the above inequality, we get

$$
0 \leq\|u-\widetilde{u}\|_{\infty} \leq 0
$$

hence, $u \equiv \widetilde{u}$. That is to say, the Equation (1.1) has a unique solution in $[0, T]$.

\section{Global existence}

To study the global existence of the solution to the Problem (1.1), we firstly need the comparison principle. Now, we begin with the definition of the sup-solution and the sub-solution to the Problem (1.1).

Definition 3.1. A function $\bar{u}(x, t) \in C(V) \times C^{1}[0, T)$ is called the sup-solution to the Problem (1.1), if it satisfied

$$
\begin{cases}\bar{u}_{t}(x, t) \geq \Delta_{\omega} \bar{u}(x, t)+\bar{u}^{p(x)}(x, t), & x \in S, t \in(0, T), \\ \bar{u}(x, t) \geq 0, & x \in \partial S, t \in(0, T), \\ \bar{u}(x, 0) \geq u_{0}(x), & x \in S .\end{cases}
$$

Similarly, we can also define the sub-solution $\underline{u}(x, t)$ to the Problem (1.1) by reversing the Inequalities (3.1).

Now, we propose the comparison principle to the Problem (1.1), which will be used to study the global existence of the solution to the Problem (1.1).

Theorem 3.2. Suppose $\bar{u}(x, t)$ and $\underline{u}(x, t)$ be the sup-solution and the sub-solution to the Problem (1.1), respectively. Then, for any $(x, t) \in V \times[0, T)$, we have $\bar{u}(x, t) \geq \underline{u}(x, t)$.

Proof. Set $v(x, t)=\underline{u}(x, t)-\bar{u}(x, t)$. Then, we have

$$
v_{t}(x, t) \leq \Delta_{\omega} v(x, t)+\underline{u}^{p(x)}(x, t)-\bar{u}^{p(x)}(x, t)
$$

for any $x \in S$ and $t \in\left[0, T_{1}\right]$, where $0<T_{1}<T$ is an arbitrary constant.

Let $v^{+}(x, t)=\max \{v(x, t), 0\}$ and then, multiplying $v^{+}$both sides of the Inequality (3.2) and integrating on $V$, we obtain

$$
\frac{1}{2}\left(\int_{x \in V}\left(v^{+}(x, t)\right)^{2}\right)_{t} \leq \int_{x \in V} \Delta_{\omega} v(x, t) v^{+}(x, t)+\int_{x \in V}\left(\underline{u}^{p(x)}-\bar{u}^{p(x)}\right) v^{+}(x, t) .
$$

For the first term of the right part of the above inequality, we have

$$
\int_{x \in V} \Delta_{\omega} v(x, t) v^{+}(x, t) \leq 0 .
$$

In fact, let $J(t)=\{x \in V: v(x, t)>0\}$ and then, we have

$$
\begin{aligned}
& \sum_{x \in V} \sum_{y \in V} v_{+}(x, t)[v(y, t)-v(x, t)] \omega(x, y) \\
& =\sum_{x \in J(t)} \sum_{y \in J(t)} v(x, t)[v(y, t)-v(x, t)] \omega(x, y)+\sum_{x \in J(t)} \sum_{y \in V \backslash J(t)} v(x, t)[v(y, t)-v(x, t)] \omega(x, y) \\
& =-\frac{1}{2} \sum_{x \in J(t)} \sum_{y \in J(t)}[v(y, t)-v(x, t)]^{2} \omega(x, y)+\sum_{x \in J(t)} \sum_{y \in V \backslash J(t)} v(x, t)[v(y, t)-v(x, t)] \omega(x, y) \\
& \leq 0 .
\end{aligned}
$$


On the other hand, for any fixed $x \in V$, we have

$$
\underline{u}^{p(x)}(x, t)-\bar{u}^{p(x)}(x, t) \leq p(x) \xi^{p(x)-1}(x, t) v(x, t),
$$

where $\xi(x, t)=\theta(x) \underline{u}^{p(x)}(x, t)+(1-\theta(x)) \bar{u}^{p(x)}(x, t)$ is bounded in $V \times\left[0, T_{1}\right]$ and $0 \leq \theta(x) \leq 1$. Now, suppose

$$
C=p^{+} \times \max _{x \in V, t \in\left[0, T_{1}\right]} \xi^{p(x)-1}(x, t) .
$$

And then, for the second term of the right part of the Inequality 3.3$)$, we also have

$$
\left.\int_{x \in V} \underline{u}^{p(x)}-\bar{u}^{p(x)}\right) v^{+}(x, t) \leq C \int_{x \in V}\left(v^{+}(x, t)\right)^{2} .
$$

Combine the Inequalities (3.3), (3.4) and (3.6), we have

$$
\left(\int_{x \in V}\left(v^{+}(x, t)\right)^{2}\right)_{t} \leq 2 C \int_{x \in V}\left(v^{+}(x, t)\right)^{2} .
$$

Furthermore, since $v^{+}(x, 0) \equiv 0$, hence, we can get $v^{+}(x, t) \equiv 0$ for any $x \in V$ and $t \in\left[0, T_{1}\right]$. By the arbitrariness of $T_{1}$, we obtain $\bar{u}(x, t) \geq \underline{u}(x, t)$ for $(x, t) \in V \times[0, T)$.

Now, we propose the global existence of the solution to the Problem 1.1) when $p^{+} \leq 1$.

Theorem 3.3. Assume that $p^{+} \leq 1$ and then, every solution to the Problem (1.1) is global.

Proof. We consider the following ODE:

$$
\left\{\begin{array}{l}
z^{\prime}(t)=z(t) \\
z(0)=\max \left\{\max _{x \in S} u_{0}(x), 1\right\}
\end{array}\right.
$$

Observe that $z^{\prime}(t)=z(t) \leq z^{p}(t)$ for any $p \leq 1$, hence, it is easy to verify that $z(t)$ is sup-solution to the problem (1.1) for $p^{+} \leq 1$ and then, since $z(t)$ is global, we have every solution to the problem (1.1) is global.

\section{Blow-up of solutions and Blow-up rate}

About the Blow-up of the equation (1.1), we have the following theorem.

Theorem 4.1. Let $u(x, t)$ be a positive solution of equation (1.1) and $p^{-}>1$, then, for a sufficiently large initial datum, there exists a finite time $T>0$ such that

$$
\|u(x, t)\|_{\infty}=+\infty
$$

when $t<T$.

Proof. Firstly, define the energy function $\eta(t)=\sum_{x \in V} u(x, t) \phi(x)$, where $\phi(x)>0$ is a eigenfunction to the first eigenvalue $\lambda_{1}$ and $\sum_{x \in S} \phi^{2}(x)=1$. And then, we get $-\Delta_{\omega} \phi(x)=\lambda_{1} \phi(x)$. Furthermore, we have

$$
\eta^{\prime}(t)=\sum_{x \in S} u_{t}(x, t) \phi(x)=\sum_{x \in S}\left(\Delta_{\omega} u+u^{p(x)}\right) \phi(x)=-\lambda_{1} \eta(t)+\sum_{x \in S} u^{p(x)} \phi(x) .
$$

Let $S_{1}=\{x \in S: u(x, t)<1\}$ and $S_{2}=\{x \in S: u(x, t) \geq 1\}$. By Jensen's inequality, we have

$$
\begin{aligned}
\sum_{x \in S} u^{p(x)} \phi(x) & =\sum_{x \in S_{1}} u^{p(x)} \phi(x)+\sum_{x \in S_{2}} u^{p(x)} \phi(x) \\
& \geq \sum_{x \in S_{2}} u^{p^{-}} \phi(x) \\
& =\sum_{x \in S_{2}} u^{p^{-}} \phi(x)+\sum_{x \in S_{1}} u^{p^{-}} \phi(x)-\sum_{x \in S_{1}} u^{p^{-}} \phi(x) \\
& \geq \sum_{x \in S} u^{p^{-}} \phi(x)-\sum_{x \in S_{1}} \phi(x) \\
& \geq \sum_{x \in S} u^{p^{-}} \phi(x)-n \\
& \geq n^{1-p^{-}}\left[\sum_{x \in S}(u \phi)\right]^{p^{-}}-n \\
& =\alpha \eta^{p^{-}}(t)-n,
\end{aligned}
$$


where $\alpha=n^{1-p^{-}}>0$ and then, we obtain

$$
\eta^{\prime}(t) \geq-\lambda_{1} \eta(t)+\alpha \eta^{p^{-}}(t)-n .
$$

Since $\alpha>0$ and the function $f(\eta)=\eta^{p^{-}}$is convex, there exists $\delta>1$ such that

$$
\alpha \eta^{p^{-}} \geq 2\left(\lambda_{1} \eta+n\right), \forall \eta>\delta .
$$

It follows easily that if $\eta(0)>\delta$, then $\eta(t)$ is increasing on with respect to a certain interval and

$$
\eta^{\prime}(t) \geq \frac{\alpha}{2} \eta^{p^{-}}(t)
$$

Thus, we have

$$
\eta(t) \geq\left(\eta^{1-p^{-}}(0)-\frac{\alpha}{2}\left(p^{-}-1\right) t\right)^{\frac{1}{1-p^{-}}}
$$

hence, we have

$$
\lim _{t \rightarrow t^{*}} \eta(t)=+\infty
$$

where $t^{*}=\frac{2}{\alpha\left(p^{-}-1\right)} \eta(0)^{1-p^{-}}$. Hence, the solution $u(x, t)$ is not global for the case $u_{0}(x)$ is large enough.

In the last context of this section, we consider the blow-up rate of the solution to the equation (1.1).

Theorem 4.2. Let $u$ be the blow-up solution to the equation (1.1), $T$ is the blow-up time and then, there exist a positive constant $C$, such that

$$
\max _{x \in S} u(x, t) \leq C(T-t)^{-\frac{1}{p^{+}-1}}
$$

where $C=\max _{x \in S}\left(\frac{1}{p(x)-1}\right)^{\frac{1}{p(x)-1}}$.

Proof. Set $d(x)=\sum_{y \in V} \omega(x, y)$ and then, the Equation (1.1) can be rewritten as

$$
u_{t}=\sum_{y \in V}(\omega(x, y) u(y, t))+u^{p(x)}-d(x) u
$$

since $u(x, t)>0$, so, we have

$$
u_{t} \geq u^{p(x)}-d(x) u .
$$

Since $p(x)>1$ and the solution blows up, thus there exists a point $x_{0} \in S$ and a time $t_{0}$, such that

$$
u^{p_{0}}\left(x_{0}, t_{0}\right)-d\left(x_{0}\right) u\left(x_{0}, t_{0}\right) \geq(1-\varepsilon) u^{p_{0}}\left(x_{0}, t_{0}\right),
$$

where $0<\varepsilon \ll 1$ and $p_{0}=p\left(x_{0}\right)$. From (4.3), the inequality holds for all times $t_{0} \leq t<T$ at this point, $x_{0}$, namely,

$$
u_{t}\left(x_{0}, t\right) \geq(1-\varepsilon) u^{p_{0}}\left(x_{0}, t\right) .
$$

Now, we consider the following ODE,

$$
\left\{\begin{array}{l}
y^{\prime}(s)=(1-\varepsilon) y^{p_{0}}(s), s>t \\
y(t)=A
\end{array}\right.
$$

where $A=(1-\varepsilon)^{-\frac{1}{p_{0}-1}} C_{0}(T-t)^{-\frac{1}{p_{0}-1}}$ and $C_{0}=\left(\frac{1}{p_{0}-1}\right)^{\frac{1}{p_{0}-1}}$ and then, we have the solution to the ODE (4.5) blows up at the time $T$. Moreover, it is easy to verify that $u\left(x_{0}, s\right)$ is a super-solution of this ODE, if there exists $t \in\left[t_{0}, T\right)$ such that $u\left(x_{0}, t\right)>A$. Hence, we have $u\left(x_{0}, s\right)>y(s)$ for all $s \in[t, T)$, so, by the 
comparison, the solution $u\left(x_{0}, t\right)$ blows up before the time $T$, which is a contradiction. Consequently, for all times

$$
u\left(x_{0}, t\right) \leq A=(1-\varepsilon)^{-\frac{1}{p_{0}-1}} C_{0}(T-t)^{-\frac{1}{p_{0}-1}} .
$$

Since $u(x, t)$ blows-up at the point $x_{0}$, thus, we have

$$
\max _{x \in S} u(x, t) \leq C(T-t)^{-\frac{1}{p^{+}-1}} .
$$

\section{Example}

In this section, we consider a graph $G_{1}$ (as shown in Figure 1), which has six nodes $x_{1}, x_{2}, \cdots, x_{6}$, where $x_{2}, x_{3}, x_{5}$ are interior and $x_{1}, x_{4}, x_{6}$ are the boundary. Moreover, we only consider the weight function $\omega \equiv 1$. Thus, the problem (1.1) can be rewritten as

$$
\left\{\begin{array}{l}
u_{t}\left(x_{2}, t\right)=u\left(x_{3}, t\right)+u\left(x_{5}, t\right)-3 u\left(x_{2}, t\right)+u^{p_{2}}\left(x_{2}, t\right) \\
u_{t}\left(x_{3}, t\right)=u\left(x_{2}, t\right)+u\left(x_{5}, t\right)-3 u\left(x_{3}, t\right)+u^{p_{3}}\left(x_{3}, t\right) \\
u_{t}\left(x_{5}, t\right)=u\left(x_{2}, t\right)+u\left(x_{3}, t\right)-3 u\left(x_{5}, t\right)+u^{p_{5}}\left(x_{5}, t\right) \\
u\left(x_{2}, 0\right)=\alpha>0 \\
u\left(x_{3}, 0\right)=\beta>0 \\
u\left(x_{5}, 0\right)=\gamma>0
\end{array}\right.
$$

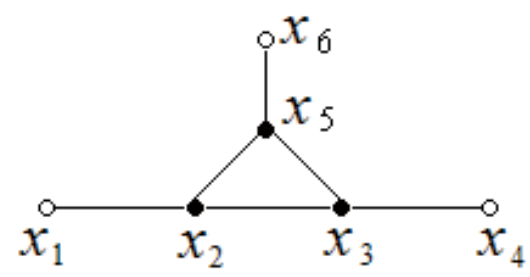

Figure 1: The graph $G_{1}$

We suppose that the exponents $p_{2}=1.4, p_{3}=1.3, p_{5}=1.2$. Moreover, the operator $-\Delta_{\omega}$ on the graph $G_{1}$ as follows:

$$
-\Delta_{\omega}=\left(\begin{array}{ccc}
3 & -1 & -1 \\
-1 & 3 & -1 \\
-1 & -1 & 3
\end{array}\right)
$$

and then, we have the first eigenvalue $\lambda_{1}=1$, the corresponding eigenvector $\phi(x)=\left(\frac{\sqrt{3}}{3}, \frac{\sqrt{3}}{3}, \frac{\sqrt{3}}{3}\right)^{T}$. By Theorem 4.1. we can choose $(\alpha, \beta, \gamma)=(3,6,9)$ and then, the solutions $u\left(x_{2}, t\right), u\left(x_{3}, t\right), u\left(x_{5}, t\right)$ will blow up. Since the Systems (5.1) is nonlinear, it is difficult to compute its analytic solutions. Thus, we consider its numerical solutions. The explicit difference scheme to the Systems (5.1) is as follows:

$$
\left\{\begin{array}{l}
\frac{u^{n+1}\left(x_{2}\right)-u^{n}\left(x_{2}\right)}{\Delta t}=u^{n}\left(x_{3}\right)+u^{n}\left(x_{5}\right)-3 u^{n}\left(x_{2}\right)+\left(u^{n}\left(x_{2}\right)\right)^{1.4} \\
\frac{u^{n+1}\left(x_{3}\right)-u^{n}\left(x_{3}\right)}{\Delta t}=u^{n}\left(x_{2}\right)+u^{n}\left(x_{5}\right)-3 u^{n}\left(x_{3}\right)+\left(u^{n}\left(x_{3}\right)\right)^{1.3} \\
\frac{u^{n+1}\left(x_{5}\right)-u^{n}\left(x_{5}\right)}{\Delta t}=u^{n}\left(x_{2}\right)+u^{n}\left(x_{3}\right)-3 u^{n}\left(x_{5}\right)+\left(u^{n}\left(x_{5}\right)\right)^{1.2} \\
u\left(x_{2}, 0\right)=3 \\
u\left(x_{3}, 0\right)=6 \\
u\left(x_{5}, 0\right)=9
\end{array}\right.
$$




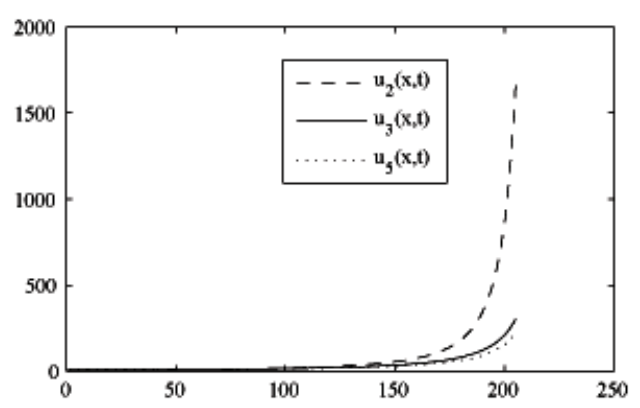

Figure 2: Blowup of the equation $\sqrt{1.1}$

where $u^{n}\left(x_{i}\right)$ denotes $u\left(x_{i}, n \Delta t\right)$ for $i=2,3,5$ and $\Delta t$ is the time step which equals 0.01 in the numerical experiment. The numerical experiment result is shown in Figure 2. From this numerical experiment, we know the solution will blow up in finite time, moreover, the blowup time strongly depends on the exponent $p^{-}$.

\section{Conclusion}

Our main theorem only considers the blow-up of the solution in the case $p(x)>1$, however, for the case $p^{-} \leq 1 \leq p^{+}$, there also exists blow-up, the further work will be needed to settle. Moreover, the critical exponent seems to depend in another way, we will consider in the further work.

\section{Acknowledgements}

The first author is supported by the Natural Science Foundation of Uygur Autonomous Region of Xinjiang(Grant No.201442137-30) and the National Natural Science Foundation of China (Grant No.11461075). The second author is supported by Scientific Research Fund of Hunan Provincial Education Department (Grant No.14B067). The authors thank the referees for their detailed comments.

\section{References}

[1] F. R. K. Chung, Spectral graph theory, CBMS Regional Conference Series in Mathematics, American Mathematical Society, Providence, (1997). 2 2.1

[2] S. Y. Chung, Critical blow-up and global existence for discrete nonlinear p-laplacian parabolic equations, Discrete Dyn. Nat. Soc., 2014 (2014), 10 pages. 1

[3] S. Y. Chung, C. A. Berenstein, w-harmonic functions and inverse conductivity problems on networks, SIAM J. Appl. Math., 65 (2005), 1200-1226. 1

[4] Y. S. Chung, Y. S. Lee, S. Y. Chung, Extinction and positivity of the solutions of the heat eqautions with absorption on networks, J. Math. Anal. Appl., 380 (2011), 642-652. 2

[5] E. B. Curtis, J. A. Morrow, Determining the resistors in a network, SIAM J. Appl. Math., 50 (1990), 918-930. 1

[6] A. Elmoataz, O. Lézoray, S. Bougleux, Nonlocal discrete regularization on weighted graphs: A framework for iamge and manifold processing, IEEE Trans. Image Prcoess., 17 (2008), 1047-1060. 1

[7] R. Ferreira, A. de Pablo, M. Préz-LLanos, J. D. Rossi, Critical exponents for a semi-linear parabolic equation with variable reaction, Proc. R. Soc. Edinburgh, 142 (2012), 1027-1042. 1

[8] J. P. Pinasco, Blow-up for parabolic and hyperbolic problems with variable exponents, Nonlinear Anal., 71 (2009), 1094-1099. 1

[9] V. Ta, O. Lézoray, A. Elmoataz, S. Schüpp, Graph-based tools for microscopic cellular image segmentation, Pattern Recognit., 42 (2009), 1113-1125. 1

[10] X. Wu, B. Guo, W. Gao, Blow-up of solutions for a semilinear parabolic equation involving variable source and positive initial energy, Appl. Math. Lett., 26 (2013), 539-543. 1

[11] Q. Xin, L. Xu, C. Mu, Blow-up for the $\omega$-heat equation with dirichlet boundary conditions and a reaction term on graphs, Appl. Anal., 93 (2014), 1691-1701. 1

[12] W. Zhou, M. Chen, W. Liu, Critical exponent and blow-up rate for the $\omega$-diffusion equations on graphs with dirichlet boundary conditions, Electron. J. Differential Equations, 2014 (2014), 13 pages. 1 